\title{
TCAP: Facilitation of learning in hypothyroid rats'
}

\author{
MICHAEL J. SCHMIDT 2 AND JOHN W. DAVENPORT \\ REGIONAL PRIMATE RESEARCH CENTER, UNIVERSITY OF WISCONSIN
}

Y-maze spatial discrimination and reversal performance of rats was facilitated by chronic administration of TCAP, a compound reported to have both RNA-stimulating and antithyroid properties. This result was found even in rats exposed to the compound sufficiently early (since conception) for its antithyroid action to produce the usual physical signs of cretinism.

The compound 1,1,3-tricyano, 2-amino, 1-propene (TCAP, also known as tricyamp, tri-a-p, and Upjohn U-9189) has been reported to have both RNA-stimulating (Egyhazi \& Hydén, 1961) and antithyroid (Ingbar, 1961; Allen et al, 1965) properties. Research on the behavioral effects of TCAP has thus far been limited to relatively short-term administration of the drug in adult rats and mice, with promising but conflicting results in simple learning and retention situations. ${ }^{3}$ In this report we present behavioral data obtained on 60and 225-day-old rats which were reared on diets containing TCAP since the time of weaning (in daily mash feedings), since birth (through mothers' milk and in mash after weaning), and since conception (through mothers' placenta and milk and in mash after weaning).

These chronic regimens produced large differences in the physical development of the Ss which will be reported in detail by Schmidt \& Allen (in preparation). Body weights at 49 days of age were reduced by TCAP to $73 \%, 47 \%$, and $38 \%$ of control rats' weights, respectively, and histological sections of the thyroid gland showed moderate to severe hyperplasia in proportion to the duration of drug exposure. Typical signs of cretinism, including reduced skeletal development of the cranium, premature closure of the epiphyseal cartilages in the long bones, and persistence of immature hair and skull proportions, were observed in the Ss having the longest and earliest drug exposure.

To assess behavioral capabilities in all three groups of Ss, we compared their performance with appropriate control Ss' in an automated $\mathrm{Y}$-maze-discrimination and reversal situation which provided measures of activity level and locomotion speed as well as learning scores. This test setting had previously produced consistently negative results in a series of unpublished studies ${ }^{3}$ on the effects of relatively acute ( 1 to 5 days) administrations of TCAP in rats and monkeys.

Method

Four groups of albino rats of the Holtzman strain received experimental treatments as follows.

Group GLW (Gestation, Lactation, Weaning). The three mothers of 16 rats comprising this group were fed ground Purina Lab Chow containing 1.5 gm TCAP per $\mathrm{kg}$ of diet beginning at approximately the day of successful mating and throughout the gestation and lactation periods. The offspring continued on this regimen after weaning until $80(\mathrm{~N}=10)$ or $225(\mathrm{~N}=6)$ days of age.

Group LW (Lactation, Weaning). The four mothers of 14 rats comprising this group were fed the same TCAP diet as in Group GLW beginning with the day of parturition, the offspring continuing on this diet after weaning until the end of the study.

Group W (Weaning). Sixteen rats obtained from the Holtzman Rat Co. at the time of weaning were immediately placed on the same TCAP diet as in Groups GLW and $\mathrm{LW}$ and continued on this diet until the end of the study.

Group C (Controls). The three mothers and 17 offspring of this group were fed ground Purina Lab Chow containing no TCAP throughout the study, which was terminated at $80(\mathrm{~N}=13)$ or $225(\mathrm{~N}=4)$ days of age in the offspring.

The rats in all groups were permitted free access to food and water except during a 12-day period (ages 4960 days or 209 to 220 days) when daily feedings were reduced so as to maintain body weights at $85 \%$ to $90 \%$ of normal levels.

The test apparatus was an automatic $\mathrm{Y}$-maze equipped with photocells, cue lights, and 45-mg food pellet dispensers located near the ends of both arms of the $Y$ and in a start-box enclosure in front of the choice point. Trials, signaled by lights and controlled by programming devices in an adjacent room, were initiated by the rat's entrance into the start-box when it was illuminated.Interruption of a photobeam in the left or right arm constituted S's choice, triggering delivering of a pellet when the choice was correct or a 3-sec. nonrewarded period in the case of an error (noncorrection procedure). Five sec. of total darkness elapsed between the end of a trial and the onset of a start-box illumination indicating availability of the next trial.

Preliminary training was given on the sixth, seventh, and eighth days of the 12-day hunger period, each day consisting of a single 10-trial session in which both left and right choices were rewarded. Reward for start-box entry was progressively decreased from 100 to $0 \%$ over the 30-trial pretraining period. A measure of activity during the pretraining sessions of the 60-day-old Ss was devised by recording the total number of photobeam interruptions (both within and between trials) made during a rat's session.

Spatial discrimination training was given in a single session on the tenth day, with the Ss trained against preferences revealed in pretraining, to a criterion of 10 
consecutive correct choices. A reversal phase was given two days later with the procedure the same except for reversal of the correct side.

\section{Results}

Mean numbers of trials to criterion in the discrimination phase were 29.7, 32.3, 26.9, and 43.1 for the GLW, LW, W, and $\mathrm{C}$ groups, respectively. After a careful check revealed no systematic differences associated with the sex, age, litter membership of the Ss, or experimenter differences, one-way analysis of variance $(F=4.86$, df $=3 / 59, p<.005)$ and subsequent NewmanKeuls tests showed all three drug-treated groups to be significantly superior $(\mathrm{p}<.05$ or $<.01)$ to the control group; no differences among the three drug groups were significant.

Median numbers of trials to criterion in the reversal phase were $34.5,37.5,60.0$, and 51.0 for the GLW, LW, $\mathrm{W}$, and $\mathrm{C}$ groups, respectively. After a significant $(\mathrm{p}<$ .05) Kruskal-Wallis test, Mann-Whitney tests showed the GLW group to be significantly superior $(p<.05)$ to the $W$ and $\mathrm{C}$ groups, with all other comparisons nonsignificant.

There were no important differences among the four groups in locomotion speed over a 33-in. distance from the start-box to the end of the chosen arm in any phase of the experiment.

Activity, as measured by the rate of photobeam crossings in pretraining, was greater in the three drug groups than in the control group. On the third day of pretraining this effect reached statistical significance $(H=8.31$, $\mathrm{p}<.05$ ), with the GLW, LW, and W groups averaging 13.9 , 12.7, and 13.2, respectively, compared with the $C$ group's 9.3 responses $/ \mathrm{min}$. The superiority of the GLW and $\mathrm{W}$ groups over the $C$ group was significant $(p<.02)$ by Mann-Whitney tests. Consistent with these findings, higher activity levels in drug-treated Ss were frequently observed by the Es in the course of handling and feeding the Ss.

\section{Discussion}

In summary, the rats reared on TCAP diets showed superior acquisition of a spatial discrimination, normal or better ability to reverse that discrimination, normal locomotion speeds, and heightened activity levels, in spite of extreme retardation of their physical development by the thyroid depressant action of this drug. That this pattern of results cannot be attributed to group differences in hunger (a potentially uncontrolled factor because of extreme weight differences among the groups) was indicated by a subsequent control experiment in which seven hungry rats $(80 \%$ of normal weight, $22 \mathrm{hr}$. deprivation) ran faster and were more active than six nonhungry rats $(90 \%, 1 \mathrm{hr}$.) but choice behavior in discrimination and reversal was unaffected by hunger level.
The present results may be contrasted with the available information on hypothyroidism which is experimentally induced by other thyroid antagonistic agents in rats. In general, this literature (Eayrs, 1960; Scow, $1946)^{3}$ indicates that thiouracil- and thiourea-induced and radiothyroidectomized hypothyroid rats are typically inferior to normal rats in the performance of simple conditioning and complex maze tasks and exhibit an overall sluggishness in terms of locomotion speed and general activity. Particularly emphasized by Eayrs $(1960, p .122)$ is the cretin rat's characteristic tendency to perseverate in the performance of an established habit in the face of environmental changes to which the normal rat will adjust; in this connection the superior reversal performance by the GLW group is especially noteworthy.

Although the obvious comparison between "TCAP" cretins and true cretins has not yet been run, enough information has accumulated to permit the conclusion that some behavioral deficits which usually accompany hypothyroidism in rats are notably absent in TCAPinduced hypothyroidism. While the mechanisms of neural development involved are by no means clear, it would appear that the usual deliterious effects of thyroxine deficiency in early developmental stages may be overcome by other effects of TCAP, such as an RNA-dependent compensatory reaction via stimulation of brain RNA metabolism.

\section{References}

Allen, J. R., Lalich, J. J., \& Schmidt, M. J. Effects of 1,1,3-tricyano 2-amino 1-propene on the thyroid and testes of the rat. Lab. Invest., 1965, 14, $1412-1418$.

Eayrs, J. T. Influence on the thyroid on the central nervous system. Brit. med. Bull., 1960, 16, 122-127.

Egyhazi, E., \& Hydén, H. Experimentally induced changes in the base composition of the ribonucleic acids of isolated nerve cells and their oligondendroglial cells. J. biophys. biochem. Cytol., 1961, 10, 403-410.

Ingbar, $\mathbf{S}$. H. The action of 1,1,3-tricyano 2-amino 1-propene (U9189 ) on the thyroid gland of the rat and its effects in human thy rotoxicosis. J. clin. Endocrin., 1961, 20, 129-139.

Scow, R. O. The retarding effect of allyl thiourea and of partial thyroidectomy at birth upon learning in the rat. J. comp. Psychol., 1946, 39, 359-370.

\section{Notes}

1. Supported by grant FR-00167 from the National Institutes of Health. We thank Mrs. Roselle Lisowe and Mr. Carl Grady for their very substantial assistance in conducting this research. 2. Now at the Department of Pharmacology, University of Missouri at Kansas City. Reprints may be obtained from either author.

3. Detailed summaries of unpublished research on TCAP and more extensive bibliographical information on hypothyrodism in rats may be obtained from the junior author. 